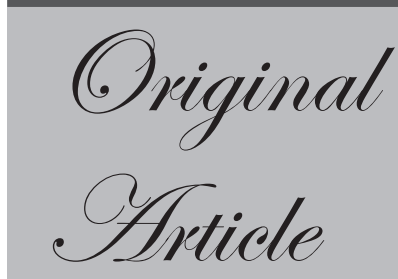

Department of Paediatrics,

Era's Lucknow Medical College

and Hospital,

Lucknow, India

Corresponding Author:

Dr Shrish Bhatnagar

Email:drshrishbhatnagar@gmail.com

\section{Bowel Habits of Healthy Indian Children Less Than Two Years of Age}

\author{
Shrish Bhatnagar, Geetika Srivastava, Ayub Ansari
}

Background: The bowel habits of children less than two years are quite varied and there is no definite hard data on stool pattern of Indian children particularly less than two years of age.

Aim: To define the normal frequency and consistency of stools of healthy Indian children between 0-24 months of age.

Methods: Parents of children aged up to 24 months were asked on a three day recall basis about their child's usual bowel habits and dietary history. Bowel habit was recorded in terms of number of stools the child passes per day, stool consistency, the age at which night bowel movements stopped, and the age of commencement of regular bowel movements. Feeding type was recorded as exclusive breast feed, mixed milk feeds or solid feeds. The bowel habits were correlated with the age and type of feeding.

Result: Children in their first six months of life had stools which were predominantly "pasty" and "runny like cream" with high and variable frequency. Beyond six months consistency was "solid" and "pasty" stools. On analyzing the combined effect of the type of milk feed and age on bowel frequency and consistency, children beyond one month of age either on exclusive breast feed or on mixed milk feed had similar frequency and consistency of stools. By one year of age more than $90 \%$ children attained regular stool pattern with no night time bowel movements.

Conclusion: This is the first report from India which describes the stool pattern of normal healthy children less than two years of age.

KEYWORDS: Bowel habits, Healthy children, Indian.

\title{
Introduction
}

Bowel habits have been defined previously in literature in terms of frequency and consistency of stools. Many researchers in the past across different regions of the world have studied the bowel habits of children and their correlation with age and type of feed. ${ }^{1,2,3,4}$ The bowel habits of children less than two years are quite varied and there is no definite hard data on the stool pattern of Indian children particularly those less than two years of 
age. Parents are unaware of what the normal frequency of stool per day and consistency of stool should be and when either of them should be called as abnormal. Thus the aim of our cross sectional study was to define the bowel habits of healthy Indian children between 0-24 months of age.

\section{Methods}

1196 children aged up to 24 months were recruited from the postnatal wards and well baby clinics of our tertiary care hospital from April 2011 to December 2014. Children were recruited into the following predefined age groups of: 0-14 days, 15-28 days, 1-3 months, 4-6 months, $7-12$ months and 13-24 months.

The following children were excluded from the study group

1. Those suffering from fever, dysentery or any concurrent illness during the time of the study.

2. Children who have had any gastrointestinal illness or any severe illness requiring medication in the last one month.

3. Those with past history of significant chronic medical illness or those who have undergone any surgery.

4. Children who were born preterm i.e. $<37$ completed weeks of gestation.

5. Those having weight and height less than the third percentile for the corresponding age.

Informed consent was obtained from the parents. After recording the socio demographic data and anthropometry of the children, parents were asked on a three day recall basis about their child's usual bowel habits and dietary history. Based on consistency, the stools were divided into 1) solid, 2) pasty/soft like peanut paste, 3) runny like cream and 4) liquid like water. The type of diet was divided into three types a) exclusive breast feed b) mixed milk Feeds: breast feed and top milk feed c) solid: children who were started on complementary feed. Before taking the opinion of parents, they were verbally given the details of each stool consistency item to avoid any misinterpretation. They were also asked about the age at which night bowel movements stopped, and the age of regular bowel movements. The interpretation of regular bowel movements was that bowel movements were nearly at the same time of the day and mothers could predict the timing of bowel passage. The research protocol was approved by our institutional ethics committee and a written informed consent was taken from parents of each enrolled child.

\section{Result}

A total of 1196 children were studied, out of which 580 $(48.5 \%)$ were males. Number of children recruited in each age group were 198(0-14 days), 200 (15-28 days), 208 (1-3 months), 200(4-6 months), 200 (7 -12 months) and 192(13-24 months). A majority of the parents were from urban setups and had free access to the toilet. The primary caregiver was the mother to $80 \%$ of children; 95\% children were born in a health care setup and had received paediatric care at birth.

\section{Dietary History}

The dietary history revealed that mixed milk (breast milk + top milk) type of feeding was the predominant method of feeding in children less than six months of age while solid feeding was the predominant type beyond that age (Table 1).

\section{Bowel Frequency with age}

The mean frequency of stool in each group is given in Figure 1. The mean frequency was high and variable in the first six months of life and subsequently became constant after six months of age. Only $17 \%$ of children less than one month passed fewer than 2 stools per day. Interestingly, there were nearly 15 to $34 \%$ of children beyond one month and less than one year of age who did not pass stools daily.

Table 1: Feeding pattern of children less than 2 years of age.

\begin{tabular}{l|l|l|l} 
& \multicolumn{3}{|c}{ Feeding type } \\
\hline Age Group & Exclusive & Mixed & Solid \\
\hline $0-14$ & 184 & 14 & 0 \\
\hline $15-28$ & 88 & 112 & 0 \\
\hline $1-3$ months & 52 & 156 & 0 \\
\hline 4-6 months & 28 & 128 & 44 \\
\hline 7-12 months & 8 & 24 & 168 \\
\hline$>13-24$ months & 0 & 8 & 184 \\
\hline
\end{tabular}




\section{Bowel Frequency with various types of diets}

It was seen that children who were on exclusive breast feed had highest frequency and variation of stools per day with a mean of $5.56+3.94$ (range $=0-20 /$ day). The mean number of stools of children who were on mixed milk feed was $4.32+3.55$ stools per day (range $=0-12$ /day) and the children who were on solid feeds had mean stool frequency of $1.64+0.9$ stools per day (range $=0-4 /$ day)

\section{Bowel Consistency with age}

"Pasty" and "runny like cream" stools were the predominant types till six months of age. Beyond six months of age most children passed "solid" and "pasty" stools.

\section{Bowel Consistency with various types of diets}

Children who were exclusively breast fed and on mixed milk feed had "pasty" or "runny like cream" stools while children on solid feeds had predominantly "solid" stools

\section{Combined effect of the type of milk feed and age on bowel frequency and consistency}

This was assessed in children up to six months of age. In the initial few days of life( $<15$ days), children on breast feeding had a higher stool frequency but after the first month of life children with mixed milk feed and exclusive breast feed had similar frequency of stools per day.

The consistency of stool was similar in both the types of feed pattern. In the first month of life it was predominantly "runny like cream" and beyond that mainly "pasty" up to six months of life.

\section{Regular bowel movements and stopping of night movements}

Half of the children had regular bowel habits by the completed age of six months and almost all (92\%) had a regular habit by one year. At an age of six and twelve months only $40 \%$ and $5 \%$ of children respectively had persistence of night bowel movements (Figure 2).

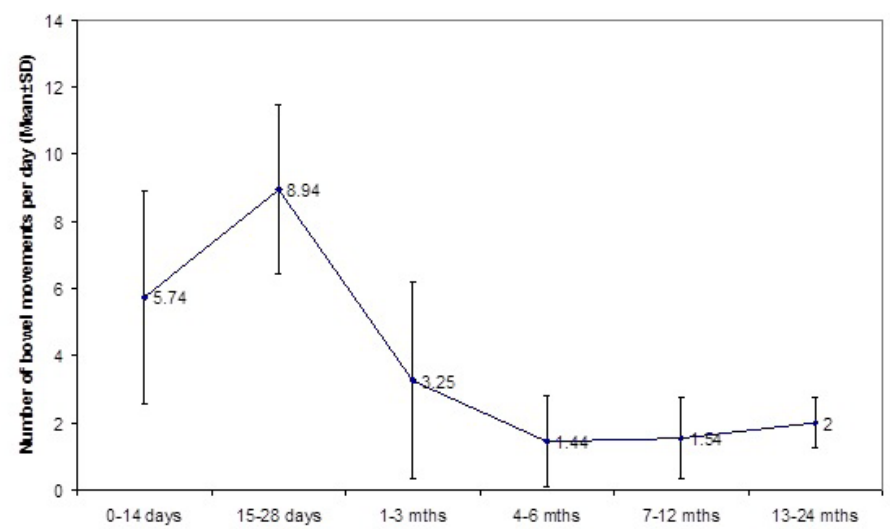

Figure 1: Mean frequency of bowel movements in each age group.

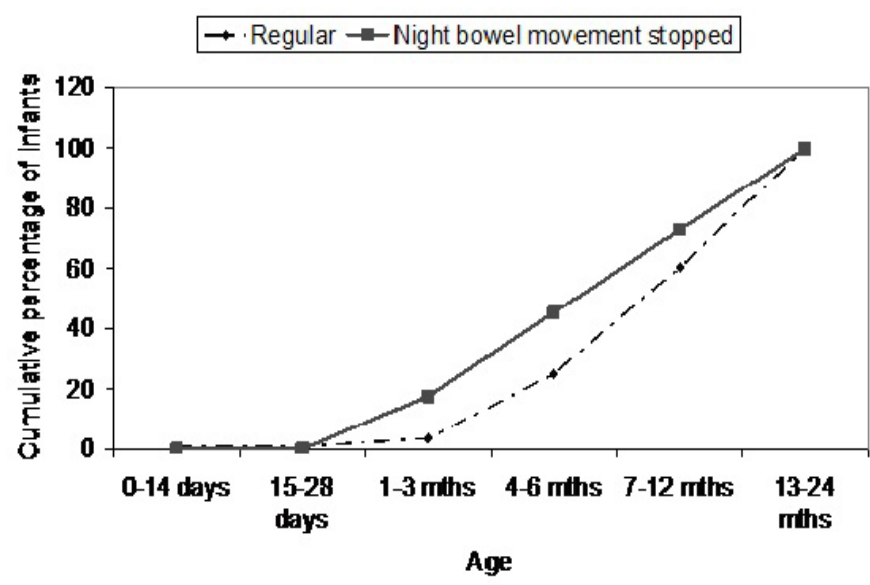

Figure 2: Pattern of regular bowel movements and stopping of night movements.

\section{Discussion}

To our knowledge, the bowel habits of healthy Indian children have not been described till now and this study tries to provide an over view of the stool pattern of young children less than two years of age and also correlate it with the type of feeds they consume. Studies in the past from various countries across globe have been published and on comparison with them, we had some interesting observations. . $^{1,2,3,4,5}$

Among children less than six months, most were on a mixed feed pattern. Only $34 \%$ were exclusively breastfed which less than the pan-India percentage of $46 \%{ }^{6}$ This is itself a cause of concern and implies an 
urgent need for launching a special program to promote exclusive breast feeding in the study region

Our study has shown that the maximum stool frequency was in the early part of life and as the child grew, the frequency decreased and almost became static by 12 months of age to 1-2 stools per day. Along with age, the type of feed given also did affect the stool frequency of children. Children on exclusive breast feeding had the highest frequency and variation of stools per day-this is an observation shared by similar studies in the past as well. ${ }^{1,4,5}$ An interesting observation was that that nearly $15-34 \%$ of children between ages of 1-12 months did not pass stools every day. This is a deviation from the Australian data which says that only $<5 \%$ of children did not pass stools every day. ${ }^{5}$ The reason for this deviation could be because of the lower percentage of exclusively breast fed infants in our study group. Human milk oligosaccharides which are prebiotic oligosaccharides have been shown to have positive effects on stool consistency and stool frequency. ${ }^{1}$

The bowel consistency also varies with age. "Pasty" and "runny like cream" stools were the predominant types till six months of age. After that "solid" and "pasty stools" were the predominant stool consistencies. These findings are similar to many studies from the past reflecting the normal physiology of gut development which has not changed over the years. ${ }^{1,3,5}$

The bowel consistency is also affected by the child's diet. Children who were exclusively breast fed and on mixed milk feed had "pasty" or "runny like cream" stools while children on solid feeds had predominantly "solid" stools. The possible explanations given for this are that the gastric emptying time for breast milk is faster than that for formula milk the difference in intestinal bacterial flora of children on different feeds and levels of circulating peptides which influence the bowel habit are different in infants on different feeds. ${ }^{7,8,9}$

Studies by Stien et al, Jaffe et al and Thompson et al have reported that the intestine of infants continues to develop after birth and bowel movements decrease during sleep. Hence it is believed that bowel movements become regular as the age of children increase. In our set of children, a similar pattern was seen and $92 \%$ had regular bowel habits and $95 \%$ had stoppage of night bowel movements by 12 months of age. ${ }^{10,11,12}$

Our study gives an overview of the stool pattern of children less than two years. However, India being an ethnically diverse country with different child rearing practices, a large multicentre pan India will give a better understand of stool pattern of normal children of the same age group.

\section{Conclusion}

To conclude, our study tries to define the stool pattern of normal healthy children less than two years of age in terms of stool frequency, consistency and its association with feeds. This study will help all paediatricians to be aware of what normal stool habits are, and this will in turn help in the better management of children and counselling of parents in their day to day practice.

\section{References}

1. Weaver LT, Ewing G, Taylor LC. The bowel habit of milkfed infants. J Pediatr Gastroenterol Nutr 1988 J; 7:568-71.

2. Fontana $\mathrm{M}$, Bianchi $\mathrm{C}$, Cataldo $\mathrm{F}$ et al. Bowel frequency in healthy children. Acta Paediatr Scand. 1989; 78:682-4.

3. Benjasuwantep B, Ruangdaraganon N. Bowel movements of normal Thai infants. Southeast Asian J Trop Med Public Health. 2009; 40:530-7.

4. Osatakul S, Yossuk P, Mo-suwan L. Bowel habits of normal Thai children. J Pediatr Gastroenterol Nutr. 1995; 20:33942.

5. Tham EBA, Nathan R, Davidson GP, Moore DJ. Bowel habits of healthy Australian children aged 0-2 years. J Paediatr Child Health. 1996; 32:504-7.

6. World Health Organization (2010a) Indicators for Assessing Infant and Young Child Feeding Practices: Part 3 Country Profiles. World Health Organization: Geneva, Switzerland.

7. Cavell B. Gastric emptying in infants fed human milk or infant formula. Acta Paediatr Scand. 1981; 70:639-41.

8. Schmitz J. McNeish AS. Development of structure and function of the gastrointestinal tract: relevance for weaning. In Ballabriga A, Rey J eds, Weaning: Why, What, and When? VeveyIRaven Press, New York. 1987; 1-30.

9. Weaver LT, Lucas A. Development of bowel habit in preterm infants. Arch Dis Child. 1993; 68(3 Spec No):31720. 
10. Stein MT. Five weeks to two months: Getting on track. In: Dixon SD. Stein MT, eds. Encounters with children: Pediatric behavior and development. 2 nd ed. St Louis: Mosby Year Book, 1992: 129-46.

11. Jaffa T, Scott S, Hendriks JH, Shapiro CM. ABC of sleep disorders. Sleep disorders in children.BMJ. 1993; 306: 640-3.

12. Thompson FM, Catto-Smith AG, Moore D, Davidson G, Cummins AG. Epithelial growth of the small intestine in human infants. J Pediatr Gastroenterol Nutr. 1998; 26: 50612. 